\title{
Clinical comparison of intravenous thrombolysis and bridging artery thrombectomy in hyperacute ischemic stroke with unknown time of onset
}

\author{
Bai Qingke ${ }^{1}$, Zheng Ping ${ }^{2}$, Zhang Jianying ${ }^{1}$, Zhao Zhenguo ${ }^{3}$
}

\author{
${ }^{1}$ Department of Neurology, Shanghai Pudong New area People's Hospital, Shanghai, \\ China \\ 2Department of Neurosurgery, Shanghai Pudong New area People's Hospital, Shanghai, \\ China \\ ${ }^{3}$ Department of Radiography, Shanghai Pudong New area People's Hospital, Shanghai, \\ China
}

Submitted: 10 August 2021; Accepted: 1 November 2021

Online publication: 9 November 2021

Arch Med Sci 2021; 17 (6): 1804-1810

DOI: https://doi.org/10.5114/aoms/143513

Copyright (c) 2021 Termedia \& Banach

\section{Abstract}

Introduction: The aim of the study was to explore the clinical efficacy and safety of intravenous thrombolysis and bridging artery thrombectomy for hyperacute ischemic stroke with unknown onset time.

Methods: One hundred and twenty-eight patients with hyperacute cerebral infarction and without a clear time of onset were randomly divided into intravenous thrombolysis $(n=66)$ and bridging artery thrombectomy groups $(n=62)$.

Results: In the intravenous thrombolysis group, 37 patients' vessels had recanalization, 32 patients' 24-hour National Institute of Health Stroke Scale (NIHSS) score improved, and 42 patients' 90-day modified Rankin Scale (mRS) score was good. In the bridging artery thrombectomy group, 62 patients' vessels had recanalization, 28 patients' 24-hour NIHSS score improved, and 38 patients' 90-day mRS score was good.

Conclusions: The benefits and adverse events between intravenous thrombolysis and bridging artery thrombectomy for ischemic stroke with unknown time of onset are similar.

Key words: large intracranial artery occlusion, cerebral infarction, magnetic resonance imaging, intravenous thrombolysis, solitaire thrombectomy.

Ischemic stroke is a disease that threatens human health seriously, which is characterized by high morbidity, mortality and disability rates. According to literature reports [1], when the hyperdense middle cerebral artery (MCA) occurs within $6 \mathrm{~h}$ of onset, $60-70 \%$ of patients may die.

Intravenous thrombolysis has been recognized as the most effective treatment for acute ischemic stroke in recent 20 years, but for stroke caused by large artery occlusion and cardiogenic embolism, the recanalization rate of intravenous thrombolysis is low, however endovascular treatment can improve the recanalization rate. Randomized trials have shown that mechanical thrombectomy with or without intravenous thrombolysis using recombinant tissue plasminogen activator (rt-PA) can improve the neurological outcomes in patients with acute stroke due to large vessel occlusion, which has been recommended by national guidelines for patients with large vessel occlusion, within $4.5 \mathrm{~h}$ of symptom onset [2]. However, about

\author{
Corresponding authors: \\ Dr. Zhao Zhenguo \\ Department \\ of Radiography \\ E-mail: zhaozhenguo1@sina. \\ com \\ Dr. Ping Zheng \\ Department of Neurosurgery \\ Shanghai Pudong \\ New Area People's Hospital \\ 490 South Chuanhuan Road \\ Shanghai 201200, China
}


$14-27 \%$ of stroke patients had an insult duration of more than $6 \mathrm{~h}$ or without a clear time window [3]. And the gold-class strategy for these stroke patients is missing. Therefore, we tried to compare the clinical efficacy and safety of intravenous thrombolysis and bridging artery thrombectomy for hyperacute ischemic stroke with unknown onset time in this study. And it is proposed that there is no difference in the benefits and ratio of adverse events for both manipulations in these patients.

Methods. Patients in the intravenous thrombolysis group combined with multi-mode magnetic resonance imaging (MRI), high signal on diffusion-weighted imaging (DWI), no high signal on T2WI and no low signal on TIWI is defined as hyperacute ischemic stroke patients? [4]. To carry out individualized rt-PA intravenous thrombolysis intervention through the pathophysiological tissue window defined by imaging, rt-PA was administered for intravenous thrombolysis. The study protocol was approved by the local Ethics Committee in Shanghai Pudong New area People's Hospital (20170223-001 on $7^{\text {th }}$ March 2017), with a waiver of informed consent due to the retrospective nature of the study. Consent for intravenous thrombolysis was obtained from all patients or related family members.

In the bridging treatment group, intravenous thrombolysis was performed immediately after multi-mode MRI showed hyperacute ischemic stroke complicated with great vessel stenosis or occlusion: human rt-PA was given at a dose of $0.9 \mathrm{mg} / \mathrm{kg}$. At the same time, interventional thrombectomy was initiated.

Observation indicators. After 24 h, 7 days, and 90 days, the neurological function was evaluated regularly, including National Institute of Health Stroke Scale (NIHSS) score (NIHSS score) and modified Rankin Scale (mRS) [5]. The effective treatment is defined as the NIHSS score decreased by $\geq$ 4 points or the symptoms disappeared completely at $24 \mathrm{~h}$ after treatment, and the good neurological function is defined as the improved Rankin scale score $\leq 2$ points after 90 days. According to the European Cooperative Acute Stroke Study II (ECASSII) standard, the hemorrhagic transformation is divided into hemorrhagic infarction $(\mathrm{HI})$ and parenchymal hematoma $(\mathrm{PH})$. Symptomatic intracranial hemorrhage $(\mathrm{SICH})$ is also defined as the deterioration of neurological function (NIHSS score $>4$ ) according to the ECASSII standard.

Statistical analysis. All the test data were analyzed by SPSS16.0 (IBM, CA, USA), and the data with a normal distribution were shown as mean \pm standard deviation indicates, and analyzed with two-sample $t$-test; $\chi^{2}$ test was carried out on the counting data. $P<0.05$ was set to be statistically significant.
Results. The comparison of baseline data between the intravenous thrombolysis group and the bridging group. There was no statistical significance in age, sex, previous history with hypertension, diabetes, atrial fibrillation ratio [6], expression level of glycosylated hemoglobin, blood glucose, triglyceride, total cholesterol, low density lipoprotein [7], blood homocysteine, fibrinogen level, preoperative NIHSS score, the Trial of Org in Acute Stroke Treatment (TOAST) [8] etiological classification of stroke and baseline characteristics (Appendix).

The comparison of functional outcomes between two groups. In the intravenous thrombolysis group, 37 patients had vascular recanalization, 32 patients had improved NIHSS score at $24 \mathrm{~h}$, and 42 patients had good mRS score after 90 days. Among 58 patients in the bridging artery thrombectomy group, 28 patients had improved NIHSS score in $24 \mathrm{~h}$, and 38 patients had good mRS score after 90 days (Table I). There was a significant difference in the recanalization rate between the two groups $(p<0.01)$, but there was no significant difference in the improvement rate of NIHSS score at $24 \mathrm{~h}$ and the good rate of neurological function in mRS score at 90 days $(p>$ 0.05). Typical cases of recanalization in the bridging treatment group and comparison of magnetic resonance imaging (MRI) and digital subtraction angiography (DSA) before and after thrombectomy in the typical case is shown in the attached figure of case 1 . Cases of vascular recanalization in the intravenous thrombolysis group are shown in the attached figure of case 2. All figures are attached as supplementary data (Appendix, Figures 1 and 2).

Comparison of cerebral hemorrhage and mortality rate between two groups. In the intravenous thrombolysis group, 6 patients had hemorrhagic transformation, 3 patients had symptomatic cerebral hemorrhage, and 4 patients died within 90 days. Twelve patients in the bridging artery thrombectomy group had hemorrhagic transformation, 6 patients had symptomatic cerebral hemorrhage, and 8 patients died within 90 days. There was no statistical difference in the rate of cerebral hemorrhage and mortality between two groups ( $p>0.05)$, as shown in Table II.

Discussion. Although mechanical thrombectomy with or without thrombolysis has been widely recommended in stroke patients within $4.5 \mathrm{~h}$ of onset, however, the treatment choice for about $14-27 \%$ of stroke patients with unknown time of onset remains elusive. Therefore, we compared two traditional manipulations called intravenous thrombolysis and bridging artery thrombectomy in these patients and found there were no advantages of one over the other. Intravenous thrombolysis and intra-arterial therapy in the acute 
Table I. Comparison of functional outcomes between the two groups

\begin{tabular}{|c|c|c|c|c|}
\hline Group & Case & $\begin{array}{c}\text { Vascular recanalization } \\
\text { rate }\end{array}$ & $\begin{array}{l}\text { Improvement rate } \\
\text { of } 24 \mathrm{~h} \text { NIHSS score }\end{array}$ & $\begin{array}{c}\text { Good rate } \\
\text { of neurological function }\end{array}$ \\
\hline $\begin{array}{l}\text { Intravenous } \\
\text { thrombolysis group }\end{array}$ & 66 & 37 (56.10\%) & $32(48.50 \%)$ & $42(63.63 \%)$ \\
\hline $\begin{array}{l}\text { Bridging treatment } \\
\text { group }\end{array}$ & 62 & $58(93.55 \%)$ & $28(45.20 \%)$ & $38(61.30 \%)$ \\
\hline$\chi^{2}$ & & 21.561 & 0.142 & 0.075 \\
\hline$P$-value & & 0.001 & 0.706 & 0.784 \\
\hline
\end{tabular}

NIHSS - National Institute of Health Stroke Scale, mRS - Modified Rankin Scale Score.

Table II. The comparison of cerebral hemorrhage transformation and mortality rate between two groups

\begin{tabular}{|lcccc|}
\hline Group & Cases & $\begin{array}{c}\text { Bleeding conversion } \\
\text { rate }\end{array}$ & $\begin{array}{c}\text { Symptomatic bleeding } \\
\text { conversion rate }\end{array}$ & Mortality rate \\
\hline $\begin{array}{l}\text { Intravenous } \\
\text { thrombolysis group }\end{array}$ & 66 & $6(9.09 \%)$ & $3(4.55 \%)$ & $4(6.06 \%)$ \\
\hline $\begin{array}{l}\text { Bridging treatment } \\
\text { group }\end{array}$ & 62 & $12(19.35 \%)$ & $6(9.68 \%)$ & $8(12.9 \%)$ \\
\hline$\chi^{2}$ & & 2.787 & 1.288 & 1.762 \\
\hline P-value & & 0.128 & 0.314 & 0.232 \\
\hline
\end{tabular}

stage of ischemic stroke are effective and relatively safe methods $[9,10]$, which mainly achieve the purpose of saving ischemic brain tissue by fast recanalization, namely clinical meaningful recanalization (CMR). Oureshi et al. [11] adjusted the potential confounding factors such as age, sex, baseline NIHSS score, systolic blood pressure, blood glucose and body temperature in the logistic regression model, found that intravenous rt-PA treatment could significantly increase the proportion of good outcome of mRS 0-1, Barthel Index $(\mathrm{BI}) \geq 95$ and Glasgow Outcome Score (GOS) 1-2 or NIHSS score $0-1$ at 90 days after onset in 91 (15\%) cases with a high density sign of the middle cerebral artery. Therefore, it is considered that intravenous rt-PA therapy is beneficial for cerebral infarction patients with large artery stenosis.

This study confirmed that the recanalization rate of stent thrombosis is higher than that of intravenous thrombolysis for patients with cerebral infarction with a narrow aorta, but there are also some problems such as high cost and limited access to professionals and equipment. In this study, the mRS scores of intravenous thrombolysis and bridging therapy for 90 days are similar, which may be related to the following factors: 1) The patients with intravenous thrombolysis are patients with hyperacute ischemic stroke screened and defined by MRI. Although there is large vessel stenosis, the rate of vascular recanalization is relatively high and the curative effect is good. Our previous studies have demonstrated the safety and effectiveness of MRI-defined intravenous thrombolysis in the hyperacute phase [12-17];
2) The time from onset to completion of treatment was shorter in simple intravenous thrombolysis than that in the bridging treatment group. Intravenous thrombolysis has effectively restored part of blood circulation and provided blood flow compensation for ischemic brain tissue to a different degree. This study indicated that although stent thrombosis has a good rate of vascular recanalization, the good rate of neurological function does not increase further, which indicates that vascular recanalization is not the only factor that determines the prognosis of patients with cerebral infarction. In conclusion, bridging artery thrombectomy based on intravenous thrombolysis did not further improve the overall prognosis as bleeding transformation, reperfusion injury and ineffective recanalization offsets the benefits of bridging therapy based on partial vascular recanalization. Therefore, stroke patients with unknown onset time might benefit from intravenous thrombolysis or bridging treatment. However, the limitation of this study needs to be addressed, such as a small sample size, single-center study and retrospective study, which requires further stratified treatment and study with larger samples to confirm.

\section{Acknowledgments}

This study was supported by funded by The Featured Clinical Discipline Project of Shanghai Pudong (PWYst2018-01) and Key Discipline Group Construction Project of Shanghai Pudong (PWZxq2017-02).

Bai Qingke and Zheng Ping contributed equally to the work. 


\section{Conflict of interest}

The authors declare no conflict of interest.

\section{References}

1. Lim J, Magarik JA, Froehler MT. The CT-defined hyperdense arterial sign as a marker for acute intracerebral large vessel occlusion. J Neuroimaging 2018; 28: 212-6.

2. Thomalla G, Simonsen CZ, Boutitie F, et al. MRI-guided thrombolysis for stroke with unknown time of onset. New Engl J Med 2018; 379: 611-22.

3. Fink JN, Kumar S, Horkan C, et al. The stroke patient who woke up. Stroke 2002; 33: 988-93.

4. Chabrier S, Ozanne A, Naggara O, et al. Hyperacute recanalization strategies and childhood stroke in the evidence age. Stroke 2021; 52: 381-4.

5. Hendrix P, Melamed I, Collins M, et al. NIHSS $24 \mathrm{~h}$ After Mechanical Thrombectomy Predicts 90-Day Functional Outcome. Clin Neuroradiol 2021; doi: 10.1007/s00062021-01068-4.

6. Wańkowicz P, Nowacki P, Gołąb-Janowska M. Atrial fibrillation risk factors in patients with ischemic stroke. Arch Med Sci 2021; 17: 19-24.

7. Hutanu A, lancu M, Dobreanu M, et al. Extended lipid profile in Romanian ischemic stroke patients in relation to stroke severity and outcome: a path analysis model. Arch Med Sci 2021; 17: 864-73.

8. Jr HPA, Bendixen BH, Kappelle LJ, et al. Classification of subtype of acute ischemic stroke. Definitions for use in a multicenter clinical trial. TOAST. Trial of Org 10172 in acute stroke treatment. Stroke 2018; 24: 35-41.

9. Chang YJ, Liu CK, Wu WP, et al. The prediction of acute ischemic stroke patients' long-term functional outcomes treated with bridging therapy. BMC Neurol 2020; 20: 22.

10. Maniskas ME, Roberts JM, Gorman A, et al. Intra-arterial combination therapy for experimental acute ischemic stroke. Clin Transl Sci 2021; DOI: 10.1111/cts.13147.

11. Mazighi M, Richard S, Lapergue B, et al. Safety and efficacy of intensive blood pressure lowering after successful endovascular therapy in acute ischaemic stroke (BP-TARGET): a multicentre, open-label, randomised controlled trial. Lancet Neurol 2021; 20: 265-74.

12. Bai $\mathrm{O}$, Zhao Z, Fu P, et al. Clinical outcomes of fast MRIbased trombolysis in wake-up strokes compared to superacute ischemic strokes within 12 hours. Neurol Res 2013; 35: 492-7.

13. Bai Q, Zhao Z, Sui H, et al. Comparison of fast MRI-based individual thrombolysis therapy for patients with superacute infarction. J Stroke Cerebrovasc Dis 2014; 23: e263-9.

14. Zhao Z, Bai Q, Sui $\mathrm{H}$, et al. Fast multimode MRI based emergency assessment of hyperacute stroke thrombolysis. Neurol Res 2013; 31: 346-50.

15. Bai $\mathrm{O}$, Zhao Z, Sui H, et al. Susceptibility-weighted imaging for cerebral microbleed detection in super-acute ischemic stroke patients treated with intravenous thrombolysis. Neurol Res 2013; 35: 586-93.

16. Bai Q, Zhao Z, Li Y, et al. The application of fast multiparametric protocol MRI-based thrombolysis with rtPA hyperacute cerebral infarction. Neurol Res 2013; 30: 344-7.

17. Bai Q, Zhao Z, Lu L, et al. Treating ischaemic stroke with intravenous TPA beyond 4.5 hours under the guidance of a MRI DWI/T2WI mismatch was safe and effective. Stroke Vasc Neurology 2019; 4: 8. 
Appendix
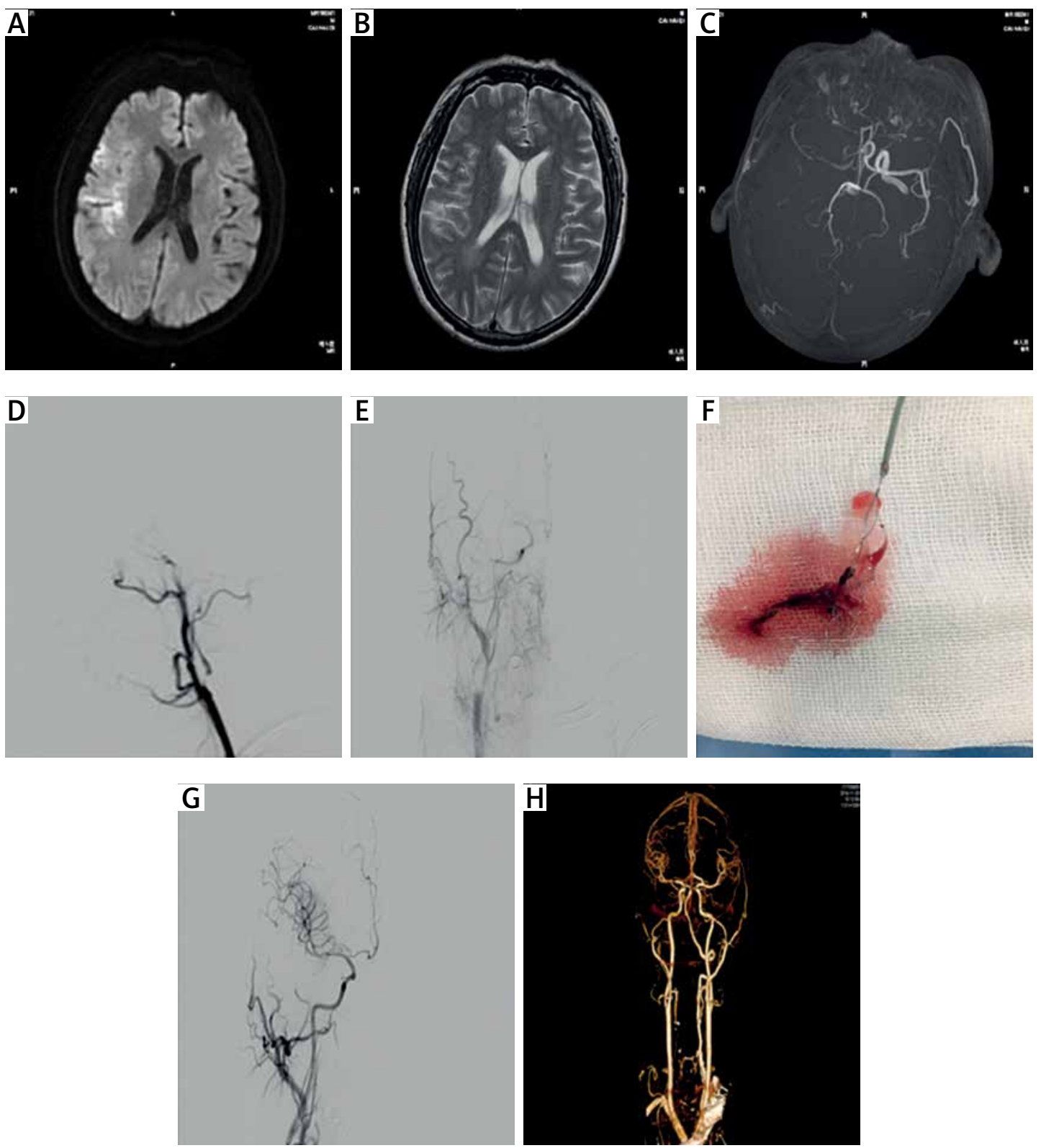

Figure 1. Left limb weakness for $3 \mathrm{~h}$, NIHSS score was 12 . The $24 \mathrm{~h}$ NIHSS score was 8 and the $90 \mathrm{~d}$ mRS score was 1 . A-C - MRI DWI, T2WI and TOF-MRA sequences before thrombolysis showed infarction in the blood supply area of the right middle cerebral artery and severe stenosis of the right middle cerebral artery. D, E-DSA showed severe stenosis or occlusion of the right internal carotid artery and right middle cerebral artery after intravenous thrombolysis. F-H - DSA and CE-MRA after thrombus removal by bridging treatment showed that the recanalization of the internal carotid artery and right middle cerebral artery was good 

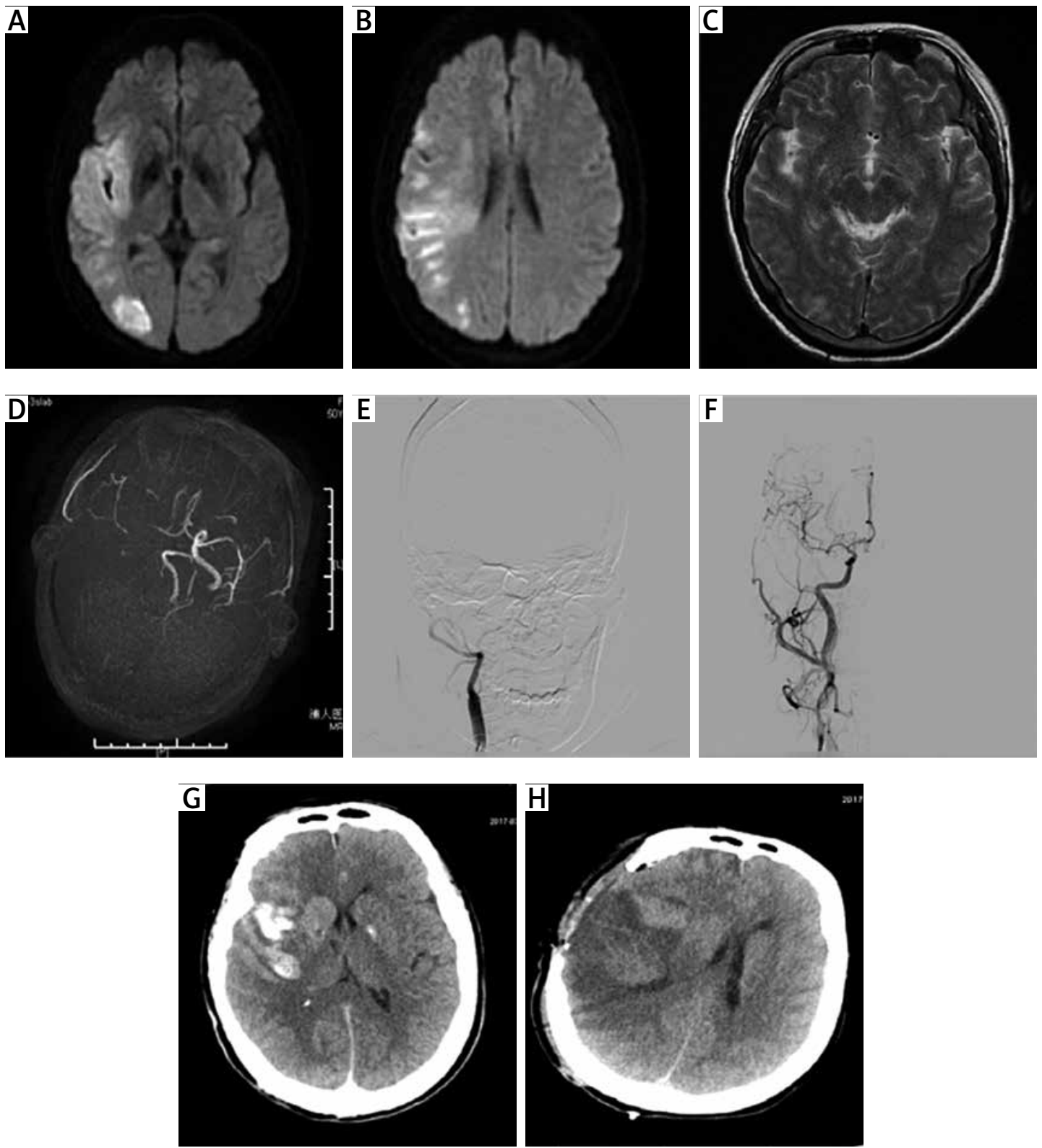

Figure 2. Case 2, left limb weakness with slurred speech for $4 \mathrm{~h}$, NIHSS score was 18, $24 \mathrm{~h}$ NIHSS score was 24 and death on $14^{\text {th }}$ day. A-D - Images before thrombolysis, DWI of the right temporal occipital lobe showed high signal intensity, but T2WI showed no obvious high signal intensity. 3D-TOF MRA showed severe stenosis of the right middle cerebral artery. E - DSA after thrombolysis, severe stenosis of the right middle cerebral artery. F-DSA after thrombectomy: right middle cerebral artery recanalization. $\mathrm{G}-\mathrm{CT}$ after thrombectomy: contrast medium remained in the right temporal lobe, and there was no obvious displacement of the ventricle and midline structure. $\mathbf{H}-36 \mathrm{~h}$ $\mathrm{CT}$, after decompression of the right temporal parietal bone flap, the right temporal lobe suffered from low density edema and high density hemorrhage, and the ventricle and midline structure shifted to the left obviously 
Table I. Comparison of baseline data between two groups

\begin{tabular}{|c|c|c|c|c|}
\hline Parameter & $\begin{array}{l}\text { Intravenous thrombol- } \\
\text { ysis group }(n=66)\end{array}$ & $\begin{array}{l}\text { Bridging group } \\
\quad(n=62)\end{array}$ & $T / U / \chi^{2}$ & $P$-value \\
\hline Age [years] & $62.2 \pm 12.8$ & $61.5 \pm 11.7$ & -1.089 & 0.290 \\
\hline Male & $45(68.2 \%)$ & $42(67.8 \%)$ & 0.166 & 0.677 \\
\hline \multicolumn{5}{|l|}{ Past diseases } \\
\hline High blood pressure & $42(67.8)$ & $40(64.5 \%)$ & 0.010 & 0.841 \\
\hline Diabetes & $15(22.7 \%)$ & $13(21.0)$ & 0.030 & 0.937 \\
\hline Atrial fibrillation & $22(33.3 \%)$ & $20(32.3 \%)$ & 0.284 & 0.579 \\
\hline \multicolumn{5}{|l|}{ Laboratory examination } \\
\hline Glycated hemoglobin & $5.7(5.4,6.8)$ & $5.8(5.4,6.7)$ & -0.243 & 0.780 \\
\hline Blood glucose $[\mathrm{mmol} / \mathrm{l}]$ & $6.4(5.3,9.5)$ & $6.3(5.4,7.2)$ & -1.028 & 0.286 \\
\hline Triglycerides [mmol/l] & $1.2(0.8,1.5)$ & $1.2(0.7,1.8)$ & -0.202 & 0.663 \\
\hline Total cholesterol [mmol/l] & $4.0(3.3,5.0)$ & $4.1(3.5,5.1)$ & -0.013 & 0.968 \\
\hline Low density lipoprotein & $2.7(2.0,3.3)$ & $2.8(2.0,3.5)$ & -0.436 & 0.760 \\
\hline Homocysteine $[\mu \mathrm{mol} / \mathrm{l}]$ & $15.8(10.8,21.3)$ & $15.0(11.8,19.8)$ & -0.576 & 0.588 \\
\hline Fibrinogen $[\mathrm{g} / \mathrm{l}]$ & $2.6(2.3,3.2)$ & $2.8(2.2,3.4)$ & -0.736 & 0.512 \\
\hline \multicolumn{5}{|l|}{ Disease severity score [points] } \\
\hline Preoperative NIHSS score & $14(12,21)$ & $14(12,20)$ & -0.030 & 0.996 \\
\hline Preoperative ASPECT score & $8(6,10)$ & $9(8,11)$ & -1.876 & 0.058 \\
\hline TOAST classification & & & 4.206 & 0.237 \\
\hline Artery atherosclerotic & $41(62.1 \%)$ & $38(61.3 \%)$ & & \\
\hline Cardiogenic embolism & $23(34.8 \%)$ & $21(33.9 \%)$ & & \\
\hline Other cause type & $0(0.0 \%)$ & $2(3.2 \%)$ & & \\
\hline Unknown causes type & $2(3.0 \%)$ & $1(1.6 \%)$ & & \\
\hline
\end{tabular}

NIHSS - National Institutes of Health Stroke Scale, ASPECT - Early CT score of Alberta stroke project, GCS - Glasgow Coma Scale. 